\title{
Mixture-of-Laplacian Faces and Its Application to Face Recognition
}

\author{
S. Noushath, ${ }^{1}$ Ashok Rao ${ }^{2}$, and G. Hemantha Kumar ${ }^{1}$ \\ ${ }^{1}$ Dept of Studies in Computer Science,University of Mysore, \\ Mysore - 570 006, India \\ nawali_naushad@yahoo.co.in \\ ${ }^{2}$ Dept of Electronics and Communication, SJ College of Engineering, \\ Mysore - 570 006, India \\ ashokrao.mys@gmail.com
}

\begin{abstract}
The locality preserving projection (LPP), known as Laplacianfaces, was recently proposed as a transformation technique of mapping which optimally preserves the neighborhood structure of the dataset. In this paper, an efficient method for face recognition called mixture-of-Laplacianfaces (or LPP mixture model) is proposed, which obtains several sets of Laplacianfaces through Expectation-Maximization (EM) learning of Gaussian Mixture Models (GMM). Experiments carried out by using this on ORL, FERET and COIL-20 indicate superior performance as compared with method based on Laplacianfaces and other contemporary subspace methods.
\end{abstract}

\section{Introduction}

The goal of dimensionality reduction algorithm is to map data points $X=$ $x_{1}, x_{2}, \ldots, x_{m}$ in $\Re^{N}$ to a subspace $\Re^{l}$, where $l \ll N$. The LPP [9] is a subspace method which also incorporates the neighborhood information while mapping the data points to a subspace. Since it preserves the neighborhood information, its classification performance is much better than other subspace approaches like PCA [6] and FLD [7. Here, we briefly outline the LPP model [9]. The main objective of LPP is to preserve the local structure of the input vector space by explicitly considering the manifold structure. The first step of this algorithm is to construct the adjacency graph $\mathbf{G}$ of $m$ nodes, such that node $i$ and $j$ are linked if $x_{i}$ and $x_{j}$ are close w.r.t each other in any of the following two conditions:

1. k-nearest neighbors: Nodes $i$ and $j$ are linked by an edge, if $i$ is among $\mathrm{k}$-nearest neighbors of $j$ or vice-versa.

2. $\epsilon$-neighbors: Nodes $i$ and $j$ are linked by an edge if $\left\|x_{i}-x_{j}\right\|^{2}<\epsilon$, where $\|\cdot\|$ is the usual Euclidean norm.

Next step is to construct the weight matrix $\mathbf{W}$, which is a sparse symmetric $m \times m$ matrix with weights $w_{i j}$ if there is an edge between nodes $i$ and $j$, and 0 if there is no edge. Two alternative criterion to construct the weight matrix: 
1. Heat-Kernel: $w_{i j}=e^{\frac{-\left\|x_{i}-x_{j}\right\|^{2}}{t}}$, if $i$ and $j$ are linked.

2. $w_{i j}=1$, iff nodes $i$ and $j$ are linked by an edge.

The objective function of LPP model is to solve the following generalized eigenvector problem:

$$
X L X^{T} a=\lambda X D X^{T} a
$$

Where $\mathbf{D}$ is the diagonal matrix with entries as $D_{i i}=\sum_{j} w_{j i}$ and $L=D-W$ is the laplacian matrix.

The transformation matrix $\mathbf{A}$ is formed by arranging the eigenvectors of Eq.(11) ordered according to their eigenvalues, $\lambda_{1}<\lambda_{2}, \ldots,<\lambda_{l}$. Thus, the feature vector $y_{i}$ of input $x_{i}$ is obtained as follows:

$$
x_{i} \rightarrow y_{i}=A^{T} \cdot x_{i} \forall i=1,2, \ldots, m
$$

Note: The $X D X^{T}$ matrix is always singular because of high-dimensional nature of the image space. To alleviate this problem, PCA is used as the preprocessing step to reduce the dimensionality of the input vector space.

\section{Mixture-of-Laplacianfaces Using GMM}

Here, mixture-of-Laplacianfaces obtained through EM learning of the GMM is presented. To implement this, we first explain the concepts of GMM [1].

\subsection{PCA Mixture Model}

The goal of this model is to partition set of all classes into several clusters and to obtain PCA transformation matrix for each cluster. Here, a class and its density function of the $N$-dimensional data $x$ is represented as $P(x)=$ $\sum_{k=1}^{K} P\left(x / k, \theta_{k}\right) P(k)$. Where $P\left(x / k, \theta_{k}\right)$ and $P(k)$ represent the conditional density and apriori probability of the $k^{\text {th }}$ cluster respectively, and $\theta_{k}$ is the unknown model parameters which is to be calculated through EM learning. The multivariate Gaussian distribution function to model $P\left(x / k, \theta_{k}\right)$ is as follows:

$$
\eta\left(x / \mu_{k}, \Sigma_{k}\right)=\frac{1}{(2 \pi)^{D / 2}|\Sigma|^{1 / 2}} e^{-\frac{1}{2}\left(x-\mu_{k}\right)^{T} \Sigma_{k}^{-1}\left(x-\mu_{k}\right)}
$$

Where $\mu_{k}$ and $\Sigma_{k}$ are the mean and covariance matrix of $k^{t h}$ cluster respectively. A distribution can be written as a linear superposition of Gaussian 2 in the form $P(x)=\sum_{k=1}^{K} \pi_{k} \eta\left(x / \mu_{k}, \Sigma_{k}\right)$. Where $\pi_{k}$ is called the mixing coefficient that is set to the fractions of data points assigned to $k^{\text {th }}$ cluster. Now log of the likelihood function is given by:

$$
\ln P(X / \pi, \mu, \Sigma)=\sum_{n=1}^{N} \ln \sum_{k=1}^{K} \pi_{k} \eta\left(x_{n} / \mu_{k}, \Sigma_{k}\right)
$$

\footnotetext{
${ }^{1}$ It is also referred as probabilistic PCA or PCA mixtures in Refs. [5] and [1].

${ }^{2}$ Note that mixture of Gaussian need not be Gaussian but Fourier of Gaussian is still a Gaussian.
} 

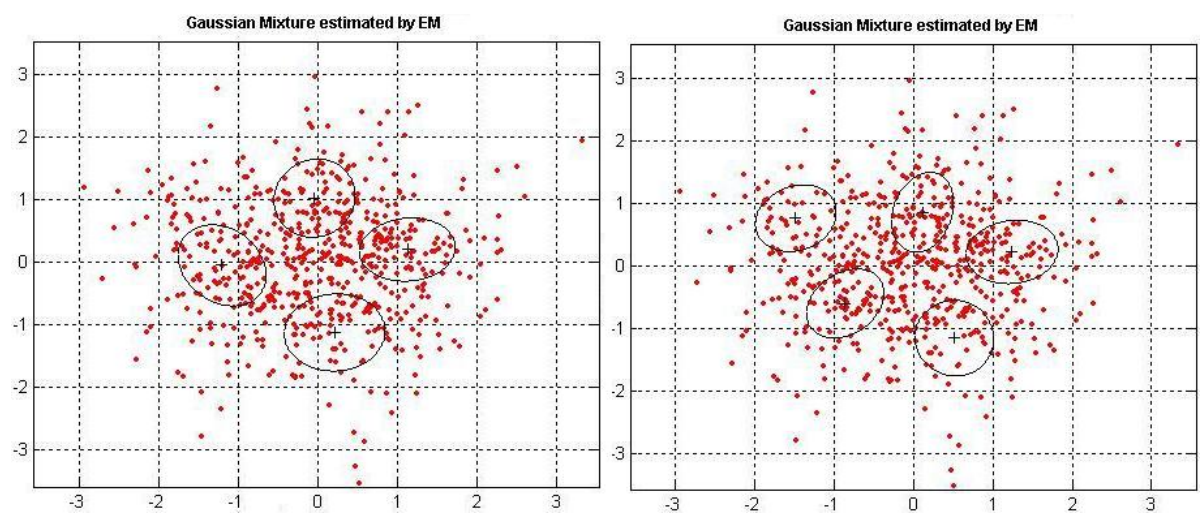

Fig. 1. Illustration of Gaussian Mixture Models

An elegant and powerful method for finding the maximum likelihood solution for GMM is called the EM algorithm [1. To find a suitable initialization for a GMM, the means $\mu_{k}$, covariances $\Sigma_{k}$ and mixing coefficients $\pi_{k}$ are initialized by running K-means clustering algorithm, which are then subsequently estimated using EM. We now alternate between the following two steps which are called Expectation(E) step and the Maximization(M) step. In the E-step, current values of the parameters are used to evaluate the posteriori probabilities given by:

$$
\xi\left(z_{n k}\right)=\frac{\pi_{k} \eta\left(x / \mu_{k}, \Sigma_{k}\right)}{\sum_{j=1}^{K} \pi_{j} \eta\left(u / \mu_{j}, \Sigma_{j}\right)}
$$

In the M-step, we use these probabilities to re-estimate the means $\mu_{k}^{\text {new }}$, covariance matrix $\Sigma_{k}^{n e w}$ and mixing coefficient $\pi_{k}^{n e w}$ respectively as follows:

$$
\begin{aligned}
\mu_{k}^{n e w} & =\frac{1}{N_{k}} \sum_{n=1}^{N} \xi\left(z_{n k}\right) x_{n} \\
\Sigma_{k}^{n e w} & =\frac{1}{N_{k}} \sum_{n=1}^{N} \xi\left(z_{n k}\right)\left(x_{n}-\mu_{k}^{n e w}\right)\left(x_{n}-\mu_{k}^{n e w}\right)^{T} \\
\pi_{k}^{n e w} & =\frac{N_{k}}{N}
\end{aligned}
$$

Each update of the parameters resulting from the E-step followed by an Mstep is guaranteed to increase the log likelihood function and the algorithm is deemed to converge when the change in the log likelihood function falls below some threshold. Fig 1 illustrates the mixture of four and five Gaussians for a data of 600 random points, where the + mark indicates the cluster means. Now, $k^{\text {th }}$ eigen value and eigen vector parameters are obtained using eigenvalue analysis $\Sigma_{k} W_{k j}=\lambda_{k j} W_{k j} \forall k=1, \ldots, K$. The PCA transformation matrix $W_{k}$ is obtained by selecting $l$ dominant eigen vectors of $k^{\text {th }}$ cluster. 


\subsection{LPP Mixture Model}

Since we obtained $K$ number of PCA transformation matrices using PCA mixture model, a feature set for each mixture is obtained in the LPP mixture model. The objective function of the proposed method now becomes as follows:

$$
X_{k} L X_{k}^{T} a_{k}=X_{k} D X_{k}^{T} a_{k} \forall k=1,2, \ldots, K
$$

Where $X_{k}$ represents $l \times n$ feature matrix of training samples obtained after the transformation through $k^{\text {th }}$ PCA mixture. The $\mathbf{D}$ and $\mathbf{L}$ matrix are obtained as mentioned previously in section 11. The transformation matrices $A_{k}=\left(a_{1}^{k}, a_{2}^{k}, \ldots, a_{l}^{k}\right)$ of LPP mixture model are formed by arranging $l$ eigenvectors of $k^{\text {th }}$ LPP mixture in Eq.(9) according to $l$ largest eigenvalues $\lambda_{1}^{k}<\lambda_{2}^{k}<\ldots,<\lambda_{l}^{k} \forall k=1,2, \ldots, K$. Using the $A_{k}$ s, features for a training sample $x$ can be obtained as follows:

$$
f_{i}^{k}=A_{k}^{T} x_{i} \forall i=1,2, \ldots, m \text { and } \forall k=1,2, \ldots, K
$$

Since there are $K$ mixtures, $K$ number of features are obtained for a unknown sample $\mathbf{I}$. To combine $K$ classification results of $\mathbf{I}$ from all the mixtures, a distance matrix is constructed and denoted by $D(I)=\left(d_{i j}\right)_{m \times K}$ where $d_{i j}$ is set to 1 if I is matched to $i^{\text {th }}$ training sample after transformation through $j^{\text {th }}$ mixture, else it is set to 0 . Consequently, the total confidence value that the sample I belongs to the $i^{t h}$ class is $T C_{I}(i)=\sum_{j=1}^{K} d_{i j}$. Finally, identity of the test sample I is computed as follows:

$$
\operatorname{Identity}(I)=\operatorname{argmax}_{i}\left(T C_{I}(i)\right) 1 \leq i \leq m
$$

\section{$3 \quad$ Experiments}

The performance of the proposed method is evaluated using three standard databases namely ORI 3 , FERET and COIL-2 4 . The nearest neighbor classifier (Euclidean distance) is used for classification and experiments are carried out on a $\mathrm{P} 43 \mathrm{GHz}$ PC with $1 \mathrm{~GB}$ RAM memory and Matlab 7.0 environment.

\subsection{Results on the ORL Database}

Two types of experiments, i.e. performance under clean and noise conditions, are carried out on this database. Our preliminary experiments suggest that the classification performance of the proposed method is impacted by the number of mixtures learned. Hence, to study the effect of number of mixtures on classification performance, we conduct an experiment by varying both number of training samples and mixtures. This result is depicted in Table迎. It is apparent

\footnotetext{
${ }^{3}$ www.uk.research.att.com/facedatabase.html

${ }^{4}$ www1.cs.columbia.edu/CAVE/research/softlib/coil-20.html

${ }^{5}$ Last row shows the results of conventional LPP method.
} 
Table 1. Recognition Accuracy(\%) for varying number of mixtures (40 dimensions)

\begin{tabular}{|c|l|l|l|l|l|l|}
\hline Number of Mixtures & \multicolumn{5}{|c|}{ Number of Training Samples } \\
\cline { 2 - 8 } & 2 & 3 & 4 & 6 & 7 & 8 \\
\hline 2 & 86.50 & 91.25 & 93.75 & 98.00 & 98.75 & 99.50 \\
3 & 88.50 & 91.50 & 94,25 & 98.50 & 99.00 & 99.50 \\
4 & 90.00 & 93.00 & 94.50 & 98.00 & 99.00 & 99.50 \\
5 & 90.50 & 93.00 & 94.00 & 98.00 & 98.75 & 99.50 \\
LPP method 9] & 82.00 & 82.25 & 89.75 & 95.00 & 96.50 & 98.25 \\
\hline
\end{tabular}

from table that, mixture of four Gaussians could be a optimal choice for competitive results and reasonable computational burden. Hence in all our subsequent experiments, we use four mixtures. Now, for comparative analysis, an experiment is conducted by training first five samples of each class. Table 2 presents the comparison of different subspace methods on recognition accuracy and running time(s). From the table it is clear that the proposed method significantly

Table 2. Comparison of different subspace methods for 40 dimensions

\begin{tabular}{|l|c|c|c|c|c|c|c|}
\hline Parameters & \multicolumn{7}{|c|}{ Methods } \\
\cline { 2 - 8 } & PCA [6 & PCA+DCT [2 & PCA+Wavelet [3 & LDA[0 & LPP[9] & PCA Mixture [5 & Proposed Method \\
\hline Accuracy(\%) & 93.25 & 93.50 & 92.50 & 92.25 & 94.50 & 95.50 & 97.00 \\
\hline Time(s) & 15.89 & 13.10 & 14.44 & 17.78 & 16.39 & 189.44 & 201.22 \\
\hline
\end{tabular}

outperformed other methods in terms of recognition accuracy. Nevertheless, the drawback of our method is the computational burden involved while EM learning of the GMM. This appears to be the case for mixture models in general.

The issue of noise modeling is crucial to check the robustness of the algorithm under real time pattern recognition and computer vision tasks. In our work, we have modeled five noise environments by using different continuous distributions in their discretized version. Using first image from each class, we generated 10

Table 3. Average recognition accuracy for different noise conditions for 25 dimensions

\begin{tabular}{|l|c|c|c|c|c|c|c|}
\hline \multirow{2}{*}{ Noise Conditions } & \multicolumn{7}{|c|}{ Methods } \\
\cline { 2 - 8 } & PCA & PCA+DCT & PCA+Wavelet & LDA & LPP & PCA Mixture & Proposed Method \\
\hline Gaussian & 81.50 & 82.00 & 68.25 & 88.00 & 87.00 & 93.50 & 95.25 \\
Salt-and-Pepper & 56.50 & 57.00 & 46.25 & 59.50 & 61.25 & 63.50 & 67.50 \\
Exponential & 31.50 & 30.50 & 78.50 & 43.00 & 51.50 & 71.00 & 73.50 \\
Weibull & 34.50 & 34.50 & 68.00 & 39.50 & 43.50 & 55.50 & 60.25 \\
Beta & 86.50 & 85.25 & 79.00 & 87.75 & 93.50 & 99.50 & 100.0 \\
\hline
\end{tabular}


Table 4. Comparing different methods under SSPP condition for 20 dimensions

\begin{tabular}{|c|c|c|c|c|c|c|c|c|}
\hline Database Size & PCA & PCA+DCT & PCA+Wavelet & Sampled-FLDA & PCA Mixture & LPP & $P C^{2} A$ & Proposed \\
\hline 20 & 100 & 100 & 100 & 100 & 100 & 100 & 100 & 100 \\
40 & 87.50 & 87.50 & 90.00 & 90.00 & 90.00 & 87.50 & 87.50 & 90.00 \\
60 & 85.00 & 90.00 & 86.66 & 88.33 & 88.33 & 84.00 & 88.33 & 88.33 \\
80 & 87.50 & 90.00 & 88.75 & 86.25 & 88.75 & 85.40 & 88.75 & 85.00 \\
100 & 85.00 & 88.00 & 91.00 & 89.00 & 83.33 & 76.00 & 87.00 & 83.33 \\
120 & 88.33 & 87.50 & 87.50 & 90.00 & 90.00 & 86.66 & 89.16 & 91.00 \\
140 & 87.14 & 89.28 & 86.42 & 88.57 & 89.28 & 86.42 & 87.14 & 89.28 \\
160 & 87.50 & 86.25 & 86.25 & 83.75 & 86.25 & 83.50 & 88.12 & 90.00 \\
180 & 86.11 & 87.22 & 88.88 & 82.22 & 86.66 & 78.88 & 86.66 & 88.88 \\
200 & 89.00 & 88.00 & 87.50 & 81.00 & 81.00 & 80.50 & 88.50 & 89.50 \\
\hline
\end{tabular}

noise images by varying the noise density from $0.1,0.2, \ldots, 1.0$. Likewise, 50 noise images are created for each class corresponding to 5 different distributions. The 2000 noisy images $(40 \times 50)$ thus created are used as test samples. First five clean samples from each class of ORL are used as training samples. Table 3 presents the average recognition accuracy (average of ten different noise densities) obtained by various algorithm. Some analysis from this experiment are:

1. The $P C A+W$ avelet method is the only robust algorithm under exponential and weibull noise conditions. Since wavelet has the advantage of both space and scale orientation, it is able to represent these noise disturbance in few scales across space and/or few space points across scales.

2. Performances of PCA and LPP mixture models are truly robust and next best performing under weibull and exponential noise because these methods are backed with Gaussian mixture models, which is known to be robust.

3. The LPP algorithm is the next best performing algorithm under all noise conditions. This we believe could be due to the utilization of the neighborhood information in this algorithm.

4. Beta noise model has had very little influence on the performance of algorithms. However, it would be interesting to see their behavior for varying values of control parameters in Beta distribution.

5. Overall the best under all conditions is the proposed method.

\subsection{Results on the FERET Database}

We have selected a partial FERET database 8 to evaluate the performance of the algorithm under single-sample-per-person (SSPP) problem. This subset database contains 400 images of 200 persons. Each person has two images (fa and $\mathbf{f b}$ ) with different facial expressions. The fa are used as gallery for training and $\mathbf{f b}$ images are used as probes for testing. Some interesting studies have been proposed exclusively to tackle this situation 810. We compare the recognition performance when number of training images is increased gradually from 20 to 200 in steps of 20 . 
Table 4 suggests that the proposed method can effectively be adapted for SSPP conditions. This is apparent when number of images in the database is increased (from 40 onwards it is as good or better than all other methods). In addition, the proposed model outperformed LPP method in all the cases by a significant margin. However, when images in the database are less(20-100), PCA+DCT and PCA+Wavelet methods outperformed other methods. This is because, for less number of training samples, the possibility of scattering of data is high (as can be seen in Fig 1). Thus GMM models work well under dense data (more number of data points), which is also the type of data where DCT/wavelets could work less better.

\subsection{Results on the COIL-20 Object Database}

This database contains 1440 gray scale images of 20 different objects, where each object contains 72 different views of varying pose angles. Initially, an ex-

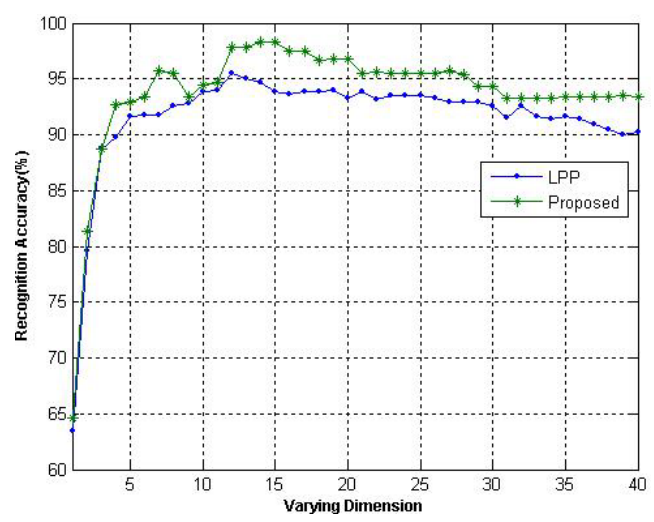

Fig. 2. Recognition accuracy for varying dimensions

periment was conducted by training first 36 samples of each object for varying dimensions. This result is depicted in Fig 2 It is quite evident that the proposed method outperformed the LPP model for all dimensions. Best recognition accuracy obtained by LPP and proposed method are $95.48 \%$ and $98.31 \%$ respectively. To make full use of the available data, another experiment was conducted by varying the number of training samples and best recognition accuracy for each case is determined. This result is shown in Table 5. It reveals that the proposed method outperformed other two methods in all the cases. This result endorses the applicability of the proposed method for object recognition.

\section{Conclusions and Future Scope}

The mixture-of-Laplacianfaces has been proposed in this paper. Unlike the conventional Laplacianface method, the proposed method obtains several sets of 
Table 5. Best Recognition accuracy(\%) on COIL-20 object database

\begin{tabular}{|c|c|c|c|c|}
\hline \multirow[t]{2}{*}{ Methods } & \multicolumn{4}{|c|}{ Number of Training Samples } \\
\hline & 12 & 18 & 24 & 36 \\
\hline$\overline{\mathrm{PCA}} 4$ & 78.89 & 81.6 & 88.13 & 93.69 \\
\hline LPP & 80.84 & 83.4 & 92.73 & 95.48 \\
\hline Proposed & 81.2 & 84.5 & 94.56 & 98.31 \\
\hline
\end{tabular}

features learned through EM algorithm of GMM. The performance of the algorithm was compared with several contemporary subspace methods under both clean and noise conditions. Besides these, we have also conducted experiments to study the performance of the proposed method under SSPP conditions and also for object images. The proposed algorithm outperformed other subspace methods under these test conditions. However, the main demerit of this algorithm is that it is computationally intensive.

Nevertheless, selecting optimal number of mixtures to yield better performance is highly subjective in nature, which deserves further study. Performance of the proposed algorithm would further improve if wavelet or DCT coefficients are used instead of raw pixel values. Performance for varying percentage of DCT coefficients or different basis of wavelets makes the study a lot more interesting. This gives lot of scope for further study and investigation.

\section{References}

1. Bishop, C.M.: Pattern Recognition and Machine Learning. Springer, Heidelberg (2006)

2. Chen, W., Er, M.J., Wu, S.: PCA and LDA in DCT domain. Pattern Recognition Letters 26, 2471-2482 (2005)

3. Chien, Chen.: Discriminant waveletfaces and nearest feature classifiers for face recognition. IEEE Transactions on Pattern Analysis and Machine Intelligence 24(12), 1644-1649 (2002)

4. Murase, H., Nayar, S.K.: Visual learning and recognition of 3D objects from appearance. International Journal of Computer Vision 14(1), 5-24 (1995)

5. Kim, H.C., Kim, D., Bang, S.Y.: Face recognition using the mixture-of-eigenfaces method. Pattern Recognition Letters 23, 1549-1558 (2002)

6. Turk, M., Pentland, A.: Eigenfaces for Recognition. Journal of Cognitive Neuroscience 3(1), 71-86 (1991)

7. Belhumeur, P., Hespanha, J., Kriegman, D.: Eigenfaces vs Fisherfaces: Recognition using Class Specific Linear Projection. IEEE Transactions on Pattern Analalysis and Machine Intelligence 19(7), 711-720 (1997)

8. Wu, J., Zhou, Z.-H.: Face recognition with one training image per person. Pattern Recognition Letters 23, 1711-1719 (2002)

9. He, X., Yan, S., Hu, Y., Niyogi, P.: Face recognition using Laplacianfaces. IEEE Transactions on Pattern Analysis and Machine Intelligence 27(3), 328-340 (2005)

10. Yin, H., Fu, P., Meng, S.: Sampled FLDA for face recognition with single training image per person. Neurocomputing 69(16-18), 2443-2445 (2006) 